\title{
Stent retriever thrombectomy combined with long-term local thrombolysis for severe hemorrhagic cerebral venous sinus thrombosis
}

\author{
YIHUA WANG $^{1}$, CUIPING ZHAO ${ }^{2}$, DEZHANG HUANG ${ }^{1}$, BIN SUN $^{1}$ and ZHIGANG WANG ${ }^{1}$
}

Departments of ${ }^{1}$ Neurosurgery and ${ }^{2}$ Neurology, Qilu Hospital of Shandong University, Qingdao, Shandong 266035, P.R. China

Received September 2, 2019; Accepted March 10, 2020

DOI: $10.3892 /$ etm.2020.9194

\begin{abstract}
Cerebral venous sinus thrombosis (CVST) is a rare disease associated with high disability and mortality rates. A subset of patients do not respond to standard anticoagulation therapy, leading to the progression of CVST with hemorrhagic stroke, which represents a major challenge for its treatment. Severe hemorrhagic (SH)-CVST is life-threatening due to large hematoma, edema and/or cerebral hernia. Anticoagulation or thrombolytic therapy alone may lead to further aggravation of the hematoma. Stent retriever thrombectomy combined with long-term local thrombolysis (SRT-LLT) has been used in certain centers for those refractory cases or patients with new intracranial hemorrhage. However, to date, no studies on SRT-LLT treatment specifically for SH-CVST have been performed. The aim of the present retrospective study was to specifically evaluate the effectiveness of SRT-LLT in SH-CVST. Between December 2013 and November 2018, SRT-LLT was performed at our center in 8 patients with hemorrhagic CVST who did not respond to intravenous anticoagulation. The clinical characteristics, results of the radiological evaluation, details on the surgical procedure and clinical outcomes were assessed. The patients were administered systemic intravenous anticoagulation as the initial treatment following admission. SRT-LLT was performed when their condition deteriorated with a high risk of a fatal outcome within a short time period. SRT-LLT was performed in 8 patients, with successful recanalization confirmed by angiography. In 4 of the patients, complete recanalization was achieved, whereas in the remaining 4 , recanalization was partial. There were no intraoperative complications. Two patients developed rebleeding after surgery, but they all gradually recovered. There were no treatment-associated fatalities. Therefore, SRT-LLT appears to be a feasible, safe and effective
\end{abstract}

Correspondence to: Professor Zhigang Wang, Department of Neurosurgery, Qilu Hospital of Shandong University, 758 Hefei Road, Qingdao, Shandong 266035, P.R. China

E-mail: neurosurgeon.cn@gmail.com

Key words: cerebral hemorrhage, cerebral venous thrombosis, thrombectomy, thrombolysis option for SH-CVST and it may be used as rescue therapy for carefully selected patients with SH-CVST.

\section{Introduction}

Cerebral venous sinus thrombosis (CVST) is a rare cause of stroke with various neurological symptoms, accounting for $\sim 1 \%$ of strokes (1). The incidence of CVST is usually underestimated, as its clinical presentation is non-specific, including headache, hemiparalysis, aphasia, seizures and even coma (2). Imaging is crucial for detecting CVST and associated complications accurately, which may affect the prognosis and the therapeutic approach $(3,4)$. Regarding the management of CVST, anticoagulation therapy is generally considered as the standard treatment. The majority of the cases have a good prognosis with standard therapy, but there is a subgroup of patients with CVST who deteriorate rapidly within a short period of time, with a potentially fatal outcome. It is necessary to detect the signs of CVST early, administer standard therapy and adjust the therapy in a timely manner. Severe clinical manifestation, an age of $>65$ years, intracranial hemorrhage ( $\mathrm{ICH})$, underlying malignancy and deep CVST are associated with higher mortality rates (5). Certain cases of large and extensive CVST are refractory to standard anticoagulation therapy and develop venous obstructive congestive ICH (6). Endovascular intra-arterial application of thrombolytic agents and/or mechanical thrombectomy for refractory cases or patients with new ICH on anticoagulation have been described over the last two decades (7). An increasing number of studies support individualized treatment for such cases with severe CVST, including endovascular thrombectomy and thrombolysis, particularly for hemorrhagic thrombosis $(8,9)$. Local contact thrombolytic therapy may effectively recanalize the thrombosed cortical veins (10). Severe hemorrhagic CVST (SH-CVST) may rapidly lead to enlargement of the hematoma, severe cerebral edema and hernia, which endanger the lives of the patients. Thrombi in the sinuses in SH-CVST must be removed as soon as possible, as they are life-threatening. Anticoagulation or thrombolytic therapy alone may lead to further aggravation of the hematoma in those SH-CVST cases. Stent retriever thrombectomy combined with long-term local thrombolysis (SRT-LLT) has been used in certain centers for those refractory cases or patients with new intracranial hemorrhage (11-13). However, to date, no studies have reported on 
SRT-LLT treatment specifically for SH-CVST. The aim of the present retrospective study was to specifically evaluate the effectiveness of SRT-LLT in SH-CVST. The present study retrospectively analyzed 10 patients with SH-CVST treated at the Qilu Hospital of Shandong University, who were initially administered anticoagulant alone. Of those cases, 8 received SRT combined with LLT when the symptoms deteriorated further. The aim of the present study was to determine whether individualized treatment with SRT-LLT may be a potential effective and safe approach for SH-CVST.

\section{Materials and methods}

Patients. The protocol of the present study was approved by the Ethics Committee of Qilu Hospital Affiliated to Shandong University (Qingdao, China). Patients or authorized relatives were informed on the risks and benefits of the operation and provided written informed consent, including consent for the publication of $\mathrm{CT}$ and digital subtraction angiography (DSA) images. A total of 29 patients with CVST (17 females and 12 males) were encountered at our center (Qilu Hospital of Shandong University, Qingdao, China) between December 2013 and November 2018, confirmed by magnetic resonance venography or DSA. Of these 29 patients, 10 had hemorrhagic CVST. Their clinical characteristics were recorded and analyzed, including age, sex, presentation, risk factors, Glasgow Coma Scale (GCS) score, location of ICH, affected venous sinus, recanalization status, outcome and complications. Systemic heparin or low-molecular-weight heparin (LMWH) subcutaneous injection was the first-line treatment for all patients after admission. LMWH sodium injection (Qilu Pharmaceutical) was usually administered at $100 \mathrm{IU} / \mathrm{kg}$ q12h. The heparin was given via intravenous injection according to the activated clotting time (ACT) if the patient had already received heparin injection at another hospital or if there was a low risk of rebleeding. SRT-LLT was performed on patients when presenting with the following: i) Failure of first-line anticoagulation; ii) rapid deterioration of consciousness or neurological impairment; and iii) significant cortical venous outflow stasis (arterio-venous circulation time $>11 \mathrm{sec}$; venous phase $>5 \mathrm{sec}$ ) (14). In the present study, 8 patients received endovascular SRT-LLT therapy.

Procedure. Interventional therapy was performed under general anesthesia. A 6-Fr and a 5-Fr introducer sheath were placed in the right femoral vein and left femoral artery, respectively. A 5-Fr catheter was placed in the left internal carotid artery. DSA revealed the CVST. Arteriovenous outflow of the cerebral circulation was detected. A 6-Fr guiding catheter was introduced into the right or left jugular bulb through the femoral vein route. Through the guiding catheter, a microcatheter was navigated into the proximal segment of the thrombosis sinus with a 0.0014-in. microwire. Subsequently, the Solitaire stent was deployed in the distal part of the thrombosed sinus and the stent was retracted after $10 \mathrm{~min}$. The manual aspiration was performed with a $50-\mathrm{ml}$ syringe, avoiding the clot of thrombus dropping through the guiding catheter during the retraction. The retraction was repeated up to 3 times. Long-term local thrombolysis was performed following thrombectomy in order to open the venous sinus and thrombosed cortical veins as much as possible to avoid further deterioration.

The 5-Fr catheter and left arterial sheath were removed. After placing an exchange microwire in the distal part of the thrombosed sinus, the catheters and introducer sheath were removed. Subsequently, the microcatheter was again passed through the sinus. An infusion pump was connected to the microcatheter. Urokinase (UK; 40,000 U/h) was infused for SRT-LLT. The microcatheter was fixed to the skin. After the procedure, the patient was returned to the Intensive Care Unit and was monitored according to the CVST observation protocol, including vital signs, GCS score and hemorrhage or thrombosis at the puncture sites. CT review was usually performed the next day after surgery, unless the condition changed. The duration of thrombolysis was no more than 7 days and was adjusted according to the improvement of the patient's condition to reduce the risk of thrombosis around the microcatheter. Oral anticoagulation treatment was administered after discontinuing local thrombolysis. Over the next 3-12 months, warfarin was orally administered. The dose of warfarin was adjusted according to the monitoring results of the international normalized ratio every 3 days.

Statistical analysis. The GCS scores at discharge were compared with those on admission by the paired t-test using SPSS v23 (IBM Corp.).

\section{Results}

Baseline characteristics. The mean age of the patients was 39 years (range, 23-65 years). A total of 4 patients $(50.0 \%)$ were females and $4(50.0 \%)$ were males. A total of 5 patients $(62.5 \%)$ presented with acute onset (within 7 days), 1 patient (12.5\%) presented with subacute onset (7-14 days) and the remaining 2 patients $(25.0 \%)$ with chronic onset ( $>14$ days). Risk factors of CVST were reported by 7 patients $(87.5 \%)$, including puerperium in 2 patients $(25.0 \%)$, diarrhea prior to onset in $2(25.0 \%)$, dehydration after strenuous physical activity in $1(12.5 \%)$, infection of the facial 'dangerous triangle' area in $1(12.5 \%)$ and coagulopathy of eosinophilia in 1 patient (12.5\%). No identifiable risk factor was detected in 1 patient (12.5\%). The symptoms at onset included headache, seizures, nausea, emesis, dizziness, papilledema, unconsciousness and hemiparalysis. CT scan in all patients revealed intracranial hemorrhage, including lobe hematoma in 4 patients $(50.0 \%)$, subarachnoid hemorrhage in 1 patient (12.5\%), multiple hemorrhages in 1 patient $(12.5 \%)$, subdural hematoma in 1 patient $(12.5 \%)$, and basilar ganglion and thalamus hemorrhage in 1 patient (12.5\%). The median GCS score was 7 (interquartile range, 3-10) at the time of the intervention. Sinus thrombosis was detected in the transverse sinus (TS) in 5 of $8(62.5 \%)$ cases, superior sagittal sinus (SSS) in $7(87.5 \%)$, sigmoid sinus (SigS) in $5(62.5 \%)$ and straight sinus (SS) in $2(25.0 \%)$ cases. In $7(87.5 \%)$ cases, $>2$ sinuses were involved. The clinical characteristics of the patients are summarized in Table I.

The initial treatment for SH-CVST with anticoagulation included administration of heparin $(3 / 8 ; 37.5 \%)$ or LMWH $(5 / 8 ; 62.5 \%)$. Furthermore, 1 patient underwent immediate craniotomy for hematoma removal and decompression in another hospital due to severe cerebral hemorrhage and 


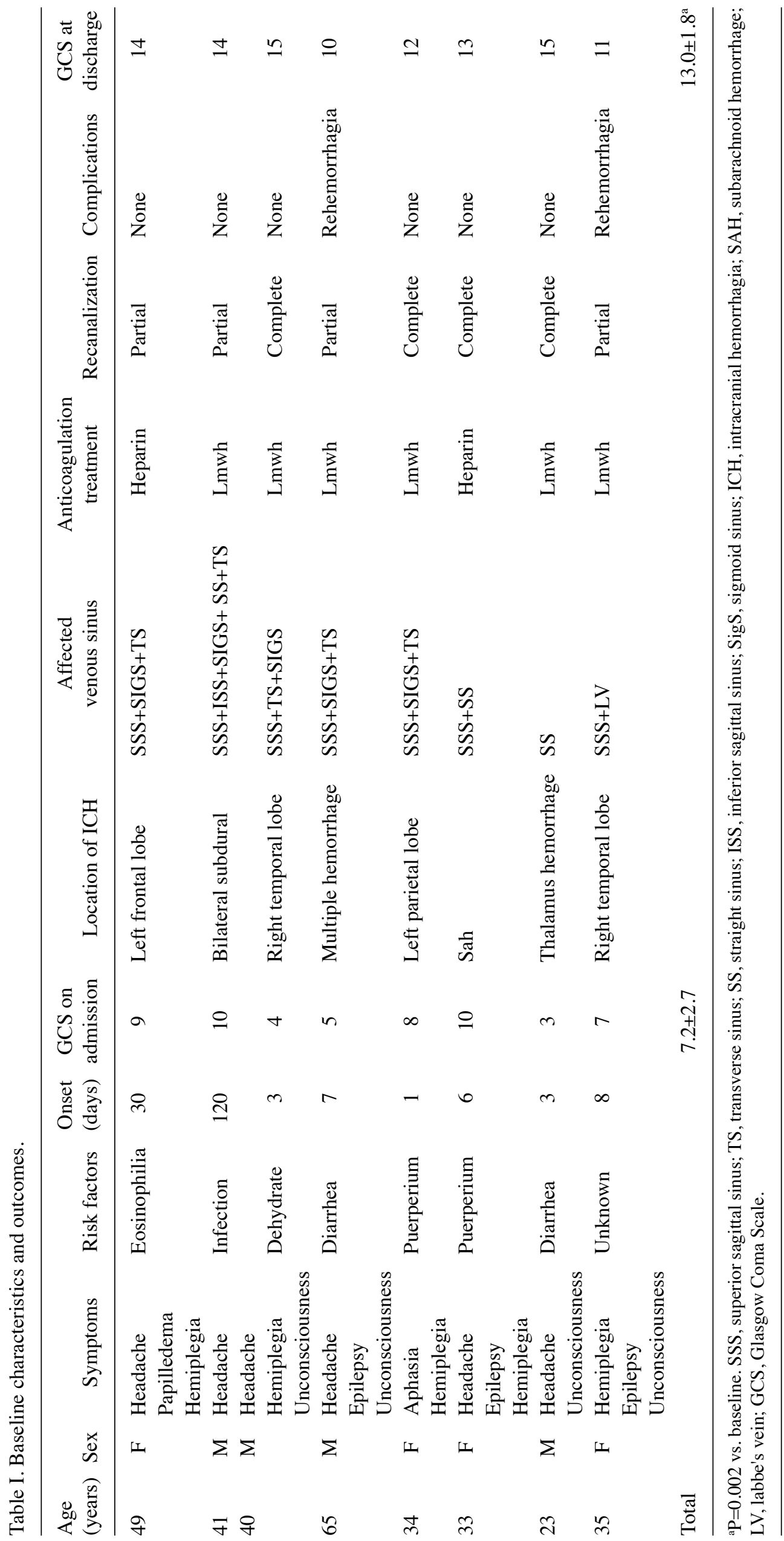



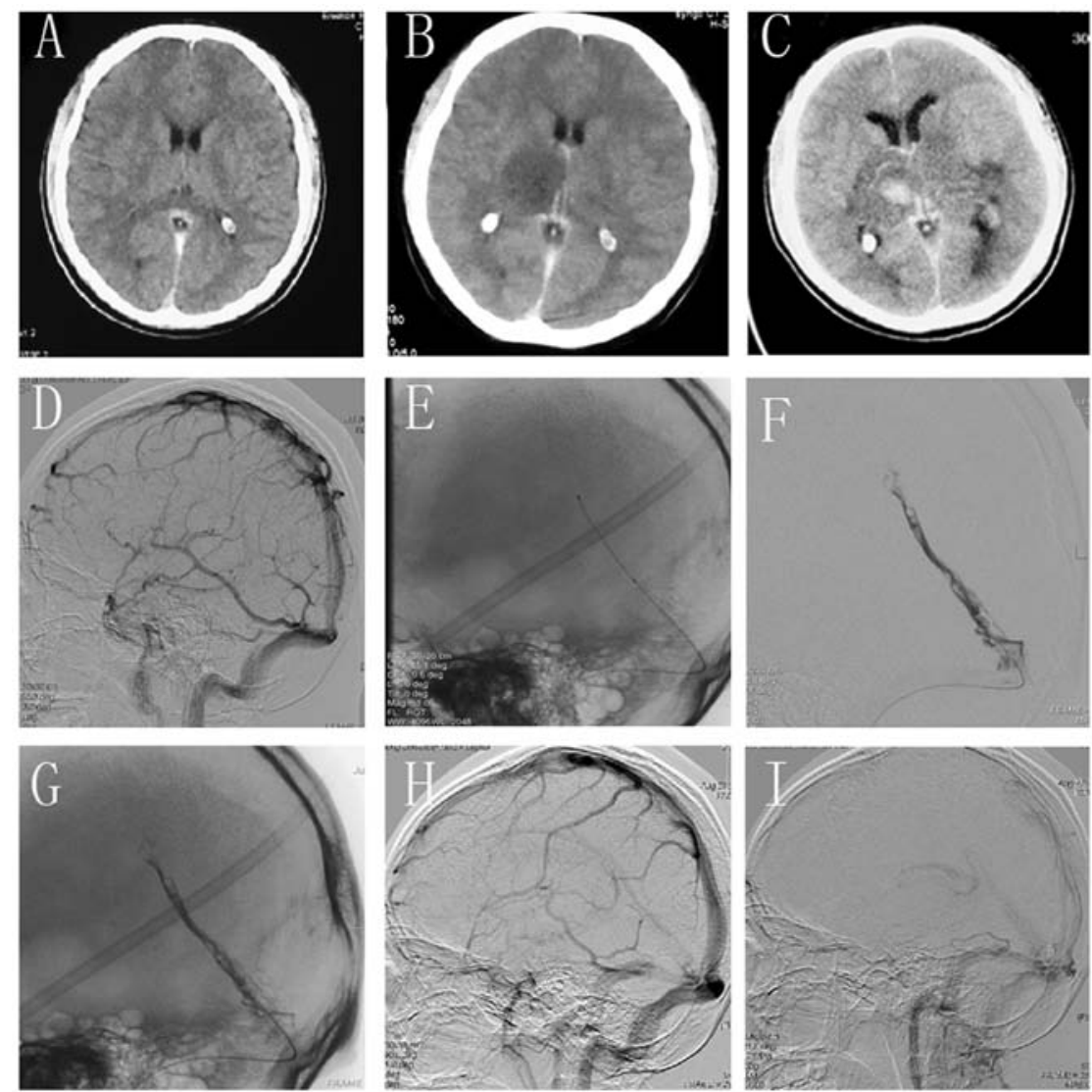

Figure 1. (A) A 23-year-old male patient presented obvious with severe headache. CT scan revealed suspected subarachnoid hemorrhage. (B) When he was transferred to our hospital, repeated CT scan revealed edema in the right thalamus area. (C) Repeated CT scan indicated a hematoma in the right thalamus with significant edema in the bilateral thalami and brain stem $24 \mathrm{~h}$ after admission. (D) DSA examination did not identify any abnormalities including aneurysm, but the presence of a thrombus in the SS was later confirmed. (E) A microcatheter was navigated and placed in the anterior portion of the SS. (F and G) DSA image showing partial recanalization of the occluded SS. (F) Subtraction image; (G) Mask image. (H and I) DSA re-evaluation revealed complete recanalization of the SS at the 1-month follow-up. CT, computed tomography; DSA, digital subtraction angiography; SS, straight sinus.

hernia. All 8 patients deteriorated or did not improve following administration of first-line anticoagulation treatment.

Endovascular SRT-LLT was performed in these cases, with successful recanalization of the occluded sinus achieved in all 8 patients; in 4 patients $(37.5 \%)$, recanalization was complete immediately after surgery, whereas the remaining 4 patients with partial recanalization achieved complete recanalization during the follow-up evaluation 3-6 months later.

No procedural associated complications were observed in these cases. ICH expanded in 2 patients (25.0\%), detected on post-interventional CT $7 \mathrm{~h}$ post-intervention. Decompressive hemicraniectomy was performed to relieve the increased intracranial pressure in 1 patient. At the time of discharge, the patient had markedly improved with moderate neurological restriction [modified Ranking scale (mRS) score, 2], without any worsening at 6 months after discharge. In another patient with enlarged hemorrhage, no increased edema or intraventricular extension occurred. The patient did not require further evacuation of the hematoma or decompressive hemicraniectomy, with moderate neurological restriction (mRS 2) at discharge and recovery 4 months post-discharge. Recurrence was defined as the presence of clinical and radiological evidence of re-thrombosis during follow-up after complete or partial recanalization. There was no recurrence of CVST in the present cohort. All patients significantly improved or were cured at discharge, and the GCS scores at discharge had significantly improved compared with those on admission. Most patients resumed their normal activities and none deteriorated or died during a follow-up period of 6 months to 3 years after discharge.

\section{Representative cases}

Case one. A 23-year-old right-handed male patient developed diarrhea after intense exercise and presented with severe headache on the following day. Scanning images of the case are provided in Fig. 1. At the local hospital, the patient was suspected to have subarachnoid hemorrhage based on non-contrast CT scans, which was later confirmed as CVST (Fig. 1A). The patient was subjected to DSA and no aneurysms or other abnormalities were identified; however, a later review confirmed the presence of a thrombus in the straight sinus (Fig. 1D). At $3 \mathrm{~h}$ after DSA examination, the patient's condition worsened and his consciousness was impaired, and he was transferred to our hospital within $2 \mathrm{~h}$. On admission, the patient was in a coma with a GCS score of 6 (E1V1M4) and had anisocoria with a 5-mm pupil and reactivity to light on the right side. He underwent another $\mathrm{CT}$ scan, which indicated edema in the right thalamus area and indirect signs of CVST (Fig. 1B). Straight sinus thrombosis was confirmed by CT and 


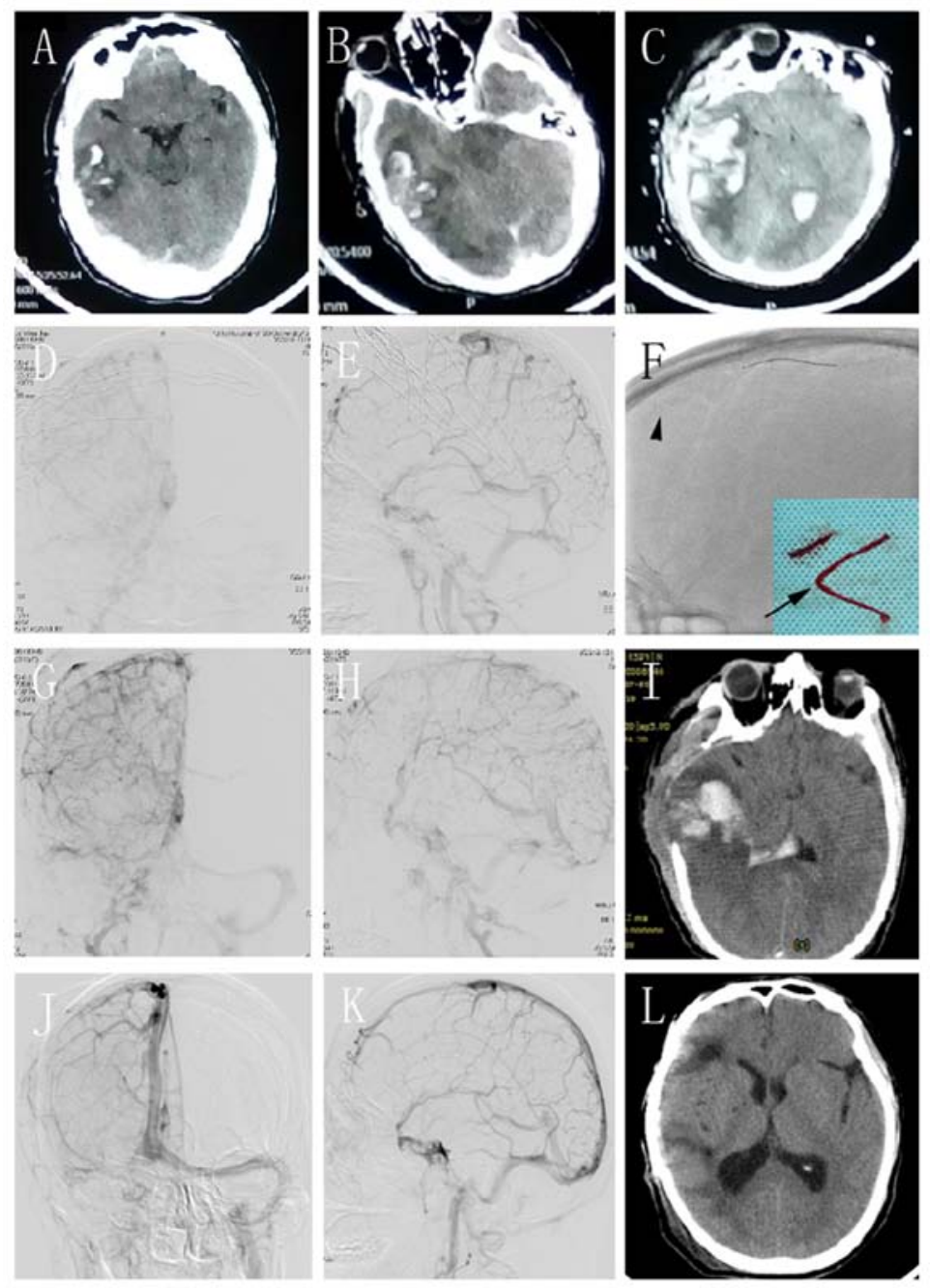

Figure 2. (A) A 47-year-old male patient presented with headache and worsened in the next day. CT scan revealed right temporal lobe hemorrhage. (B) Repeat CT scan revealed increased hematoma and cerebral herniation $4 \mathrm{~h}$ after first CT scan. (C) Repeat CT scan revealed re-bleeding after craniotomy. (D and E) The (D) positive and (E) lateral DSA sinus phase image of the right side demonstrated extensive severe CVST. (F) Stent retriever thrombectomy was performed. The arrow indicates the solitaire FR stent deployed in the occluded SSS. The arrowhead indicates the clots that were removed. (G and H) DSA revealed partial recanalization of the occluded sinus. (I) CT scan revealed absorption of the hematoma and a decrease of the edema on the 6th day after the operation. ( $\mathrm{J}$ and K) DSA revealed complete recanalization of the SSS with normal arteriovenous blood flow in the left TS, but with partial occlusion of the right TS and sigmoid sinus at the 4-month follow-up. (L) The 4-month follow-up CT scan was normal, apart from the cranioplasty. CT, computed tomography; DSA, digital subtraction angiography; CVST, central venous sinus thrombosis; SSS, superior sagittal sinus; TS, transverse sinus.

DSA (Fig. 1B and D). The patient was administered LMWH as a subcutaneous injection and further supportive medications; $24 \mathrm{~h}$ later, however, he progressively deteriorated and the GCS score decreased to 3 (E1V1M1). On repeated CT scans, the patient had hematoma in the right thalamus, with significant edema in the bilateral thalami and brain stem (Fig. 1C). The patient then received intervention therapy.

A 6-Fr guiding catheter was introduced into the right internal jugular vein. The microcatheter (Rebar 27) was then inserted into the distal part of the thrombosed SS (Fig. 1E). Next, a 6x30-mm Solitaire FR stent was deployed in the thrombosed segment and the stent was retracted into the sheath 10 min later; however, the sinus was not recanalized. The retraction was repeated three times and 300,000 IU of UK were injected for total thrombolysis. On DSA, partial recanalization of the thrombosed SS was achieved (Fig. 1F). Over the exchange wire, the Rebar 27 microcatheter was redelivered to the very distal part of the SS with a $300-\mathrm{cm}$ exchange wire.
The infusion pump was then connected to the microcatheter. UK $(40,000 \mathrm{IU} / \mathrm{h})$ was infused by the pump for SRT-LLT. On the second day after the operation, the patient's state improved and he was able to follow the doctor's instructions. The patient improved significantly every day over the following days. He was fully conscious and his physical activity was good on the 7 th postoperative day, with a GCS score of 15 . Thrombolysis via the microcatheter was stopped and oral anticoagulant therapy was continued. At the 1-month follow-up, the patient had recovered completely (Fig. 1I) and DSA re-evaluation revealed complete recanalization of the SS (Fig. $1 \mathrm{G}$ and $\mathrm{H}$ ).

Case two. A 47-year-old right-handed man presented with a headache after $14 \mathrm{~h}$ of driving, which worsened the next day. The head CT examination performed at the local hospital revealed hemorrhage in the right temporal lobe (Fig. 2A). The patient was unconscious with anisocoria in $4 \mathrm{~h}$ and the repeat $\mathrm{CT}$ revealed an increase in the size of the hematoma, with 
midline shift and cerebral herniation (Fig. 2B). The patient underwent craniotomy for hematoma removal and decompression. However, his condition deteriorated and the repeat CT scan revealed re-bleeding after surgery (Fig. 2C). The patient was transferred to our hospital with a GCS score of 7 (E1V2M4). The CT image was carefully reviewed and CVST was confirmed as the cause of the hemorrhage. The patient was administered anticoagulants and other supportive medications. However, the treatment was unsuccessful and the GCS score decreased to 4 (E1V1M2). The patient received intervention therapy. DSA revealed extensive severe CVST, involving the SSS, bilateral TS, right SigS and right internal jugular (Fig. 2D and E). A 6-Fr guiding catheter was used, but any attempts to pass it through the occluded right internal jugular vein were unsuccessful. Therefore, navigation into the left side was pursued. A microcatheter (Rebar 27) was placed to reach the distal part of the SSS. The Solitaire FR stent was deployed and subsequently retracted $10 \mathrm{~min}$ later (Fig. 2F; arrow). Several of the clots were successfully removed (Fig. 2F; arrowhead). Angiography revealed that the occluded sinus was partially recanalized with residual thromboses and the arteriovenous transit time was shortened (Fig. 2G and H). The patient received the same procedure as the previous case. UK $(40,000 \mathrm{IU} / \mathrm{h})$ was infused for SRT-LLT. The repeat CT revealed absorption of the hematoma and decrease of the edema on the 6th day after the operation (Fig. 2I). The thrombolysis was discontinued and the patient recovered with a GCS score of 15 . He was discharged with fully restored consciousness and no neurological dysfunction 14 days later.

At the 4-month follow-up, the patient's neurological function was normal. The findings on the CT scan were normal, except for the cranioplasty (Fig. 2L). The DSA revealed complete recanalization of the SSS, with normal arteriovenous blood flow in the TS on the left side but partial occlusion on the right side (Fig. 2J and $\mathrm{K}$ ). He was prescribed oral anticoagulants for 1 year.

\section{Discussion}

CVST is a rare disease with a potentially fatal outcome. During recent decades, its incidence has markedly increased, which may be attributed to advances in imaging techniques (15). However, CVST may not be timely diagnosed, as its clinical presentations are atypical and non-specific (2). Non-contrast CT scan is only able to reveal indirect signs of CVST and has low sensitivity and specificity (16). A number of the patients in the present study were not accurately diagnosed with CVST at their primary hospital; they were initially misdiagnosed, e.g. with postpartum reaction, fatigue or simple diarrhea, which led to rapid aggravation of the symptoms, ICH and life-threatening complications due to the absence of specific treatment. The timely and correct treatment for SH-CVST remains controversial. Systemically administered anticoagulation with heparin (unfractionated or LMWH) is the standard first-line treatment for CVST, even in patients with an $\mathrm{ICH}$ at baseline (17). However, a subset of patients are refractory to standard anticoagulation therapy, leading to complications, including ischemic and hemorrhagic stroke, cerebral edema and even death (3). Viegas et al (18) reported that $88 \%$ of patients with CVT recovered following systemic thrombolysis, but 2 patients died due to intracranial hemorrhage. Systemic thrombolytic therapy, particularly high-dose thrombolysis, still poses a risk of inducing bleeding. Gala et al (19) recommend thrombolytic therapy after failure of anticoagulant therapy, followed by interventional thrombectomy therapy if the thrombolysis treatment was unsuccessful. Guo et al (20) reported that 73\% of patients with CVST had a good outcome of intrasinus thrombolytic treatment. The present study included 10 patients with hemorrhagic CVST who were first treated with anticoagulation, 8 of whom deteriorated rapidly in the first $24-48 \mathrm{~h}$. It was decided to perform intervention therapy directly when the patients deteriorated further even after receiving anticoagulation treatment. It was considered that systemic thrombolysis may induce rebleeding in these hemorrhagic patients, and also that thrombolysis may not act rapidly enough in patients with a life-threatening condition. Therefore, thrombectomy combined with local thrombolysis was performed to reduce the risk of rebleeding.

Endovascular mechanical thrombectomy is considered to be an effective treatment in addition to systemic anticoagulation in severe CVST, due to the potential immediate reestablishment of antegrade venous flow, based on the success of mechanical clot retrieval in the arterial vasculature for acute ischemic large-vessel occlusion stroke (21). SRT-LLT achieved complete recanalization in $50 \%$ and partial recanalization in $50 \%$ of the SH-CVST patients who did not respond well to anticoagulation therapy. Although there were two cases of rebleeding after the intervention, this was attributed to increased congestion hemorrhage due to the obstruction of venous reflux by the thrombosis rather than procedural complications, including injury of the vessels or the side effects of local thrombolysis. Styczen et al (8) reported that of 14 patients with CVST undergoing thrombectomy, recanalization was successful in $86 \%$ of cases and complete recanalization was achieved in $29 \%$. In the present study, complete recanalization was achieved in 50\% of the cases due to combined therapy. Rapid and complete recanalization may reverse the life-threatening condition in patients with SH-CVST.

Previous studies reported complete recanalization in $87 \%$ and partial recanalization rates in $6 \%$ of patients with severe CVST who were treated with a combination of anticoagulation and intervention $(16,22)$. Good recanalization rates were obtained in certain centers with continuous local thrombolysis after mechanical thrombectomy (23-26). However, they did not specifically focus on cases with SH-CVST. The lower rate of complete recanalization in the present study was probably due to the delayed diagnosis, and there was more severe thrombosis in the obstructed sinuses compared with that in other patients with non-hemorrhagic CVST. Acute clots are easier to completely remove, as they primarily consist of erythrocytes, with less fibrin cross-linking and collagen deposition. Subacute or chronic thrombi are more difficult to compress and dissolve due to the higher collagen and cross-linked fibrin content (27). In the present study, multiple sinuses were affected, complete recanalization was not achieved by thrombectomy alone and long-term local thrombolysis was required.

For cases of CVST with a life-threatening severity, endovascular intervention, including mechanical thrombectomy, thromboembolic aspiration or balloon venoplasty, with or without local thrombolysis, are alternative treatment methods $(22,28)$. Local thrombolysis during endovascular 
intervention was first described in 1988 by Scott et al (29), and it gradually became more popular and more frequently practiced in the clinical setting. UK is the most frequently used agent (30). In most of the previous studies, it was used at doses of 250,000-1,000,000 $\mathrm{U}$ in one session during endovascular intervention $(11,13,29,31)$, or as a continuous local infusion over several days. Local thrombolysis further dissolves residual thrombi and cortical venous thrombosis (10).

As showcased in the second representative case of the present study, it is dangerous to perform craniotomy blindly when encountering an atypical cerebral hemorrhage. Intracerebral hemorrhage or edema in the internal capsule, thalami or basal ganglia highly suggests the possibility of deep CVST $(4,32)$. Just as in the second case, it led to rebleeding. Zuurbier also reported that new hemorrhagic lesions were identified on post-decompressive craniectomy and/or hematoma evacuation in 4 of 10 patients and tended to be associated with poor outcomes (33). However, it may be recommended that all attempts should be made to treat severe cases by more progressive methods in addition to conservative treatment. Thrombectomy and thrombolysis may be performed even in cases of SH-CVST. According to the study by Kulcsár et al (11), thrombectomy and long-term thrombolysis are complementary without increasing the risk of rebleeding.

There were certain limitations to the present study. First, it was not a randomized controlled trial and the number of cases was too small for a conclusive statistical analysis. It is most likely due to the low incidence of hemorrhagic CVST that there are no large-scale prospective clinical studies on interventional therapy for SH-CVST.

Almost all cases of SH-CVST deteriorated following an initial mild presentation. It is crucial to evaluate the signs and the risk factors of CVST and adjust therapy in a timely manner to avoid progression to a life-threatening condition.

In conclusion, SRT-LLT is feasible, safe and effective for SH-CVST. It may be applied as a rescue therapy for the patients with SH-CVST who are refractory to standard medical treatment.

\section{Acknowledgements}

Not applicable.

\section{Funding}

The present study was supported by a grant from Shandong Province Outstanding Young Scientists Fund (grant no. BS2012YY012).

\section{Availability of data and materials}

The datasets used and/or analyzed during the current study are available from the corresponding author on reasonable request.

\section{Authors' contributions}

YW and ZW designed and performed the current study. CZ and DZ performed analyses. YW, ZW, DZ and BS administered treatment. All authors read and approved the final manuscript.

\section{Ethics approval and consent to participate}

The protocol of the present study was approved by the Ethics Committee of Qilu Hospital Affiliated to Shandong University (Qingdao, China). Patients or authorized relatives were informed on the risks and benefits of the operation and provided written informed consent.

\section{Patient consent for publication}

Informed consent for publication of images was obtained from all subjects or their relatives.

\section{Competing interests}

The authors declare that they have no competing interests.

\section{References}

1. Silvis SM, de Sousa DA, Ferro JM and Coutinho JM: Cerebral venous thrombosis. Nat Rev Neurol 13: 555-565, 2017.

2. Linn J,Ertl-Wagner B, Seelos KC, Strupp M, Reiser M, Bruckmann H and Brüning R: Diagnostic value of multidetector-row CT angiography in the evaluation of thrombosis of the cerebral venous sinuses. AJNR Am J Neuroradiol 28: 946-952, 2007.

3. Saposnik G, Barinagarrementeria F, Brown RD Jr, Bushnell CD, Cucchiara B, Cushman M, deVeber G, Ferro JM and Tsai FY; American Heart Association Stroke Council and the Council on Epidemiology and Prevention: Diagnosis and management of cerebral venous thrombosis: A statement for healthcare professionals from the American Heart Association/American Stroke Association. Stroke 42: 1158-1192, 2011.

4. Leach JL, Fortuna RB, Jones BV and Gaskill-Shipley MF: Imaging of cerebral venous thrombosis: Current techniques, spectrum of findings, and diagnostic pitfalls. Radiographics 26 (Suppl 1): S19-S43, 2006.

5. Nasr DM, Brinjikji W, Cloft HJ, Saposnik G and Rabinstein AA: Mortality in cerebral venous thrombosis: Results from the national inpatient sample database. Cerebrovasc Dis 35: 40-44, 2013.

6. Capecchi M, Abbattista M and Martinelli I: Cerebral venous sinus thrombosis. J Thromb Haemost 16: 1918-1931, 2018.

7. Ilyas A, Chen CJ, Raper DM, Ding D, Buell T, Mastorakos P and Liu KC: Endovascular mechanical thrombectomy for cerebral venous sinus thrombosis: A systematic review. J Neurointerv Surg 9: 1086-1092, 2017.

8. Styczen H, Tsogkas I, Liman J, Maus V and Psychogios MN: Endovascular mechanical thrombectomy for cerebral venous sinus thrombosis: A single-center experience. World Neurosurg 127: e1097-e1103, 2019.

9. Mokin M,Lopes DK, Binning MJ, Veznedaroglu E, Liebman KM, Arthur AS, Doss VT, Levy EI and Siddiqui AH: Endovascular treatment of cerebral venous thrombosis: Contemporary multicenter experience. Interv Neuroradiol 21: 520-526, 2015.

10. Mortimer AM, Bradley MD, O'Leary S and Renowden SA: Endovascular treatment of children with cerebral venous sinus thrombosis: A case series. Pediatric Neurol 49: 305-312, 2013.

11. Kulcsár Z, Marosfoi M, Berentei Z and Szikora I: Continuous thrombolysis and repeated thrombectomy with the Penumbra System in a child with hemorrhagic sinus thrombosis: Technical note. Acta Neurochir (Wien) 152: 911-916, 2010.

12. Guo XB, Song LJ and Guan S: Endovascular treatment of chronic, recurrent headache secondary to chronic cerebral venous sinus thrombosis. J Stroke Cerebrovasc Dis 23: 560-563, 2014.

13. Chen C, Li X, Huang L, Zhang J, Chen S, Ye H, Ye Q, Zhang T, Zhang X, Chen Z, et al: Mechanical thrombectomy with intraoperative local thrombolysis versus mechanical thrombectomy with continuous thrombolysis for treatment of cerebral venous sinus thrombosis: A systematic review of 82 cases. World Neurosurg 125:489-497.e14, 2019

14. Chen C, Wang Q, Li X, Lu Z, He J, Fang Q, Ke X, Duan C and Li T: Stent retriever thrombectomy combined with local thrombolytic therapy for cerebral venous sinus thrombosis: A case report. Exp Ther Med 14: 3961-3970, 2017. 
15. Coutinho JM, Zuurbier SM, Aramideh M and Stam J: The incidence of cerebral venous thrombosis: A cross-sectional study. Stroke 43: 3375-3377, 2012

16. Dmytriw AA, Song JSA, Yu E and Poon CS: Cerebral venous thrombosis: State of the art diagnosis and management. Neuroradiology 60: 669-685, 2018.

17. Ferro JM, Bousser MG, Canhao P, Coutinho JM, Crassard I, Dentali F, di Minno M, Maino A, Martinelli I, Masuhr F, et al: European Stroke Organization guideline for the diagnosis and treatment of cerebral venous thrombosis-endorsed by the European Academy of Neurology. Eur J Neurol 24: 1203-1213, 2017.

18. Viegas LD, Stolz E, Canhão P and Ferro JM: Systemic thrombolysis or cerebral venous and dural sinus thrombosis: A systematic review. Cerebrovasc Dis 37: 43-50, 2014.

19. Gala NB, Agarwal N,Barrese J, Gandhi CD and Prestigiacomo CJ: Current endovascular treatment options of dural venous sinus thrombosis: A review of the literature. J Neurointerv Surg 5: $28-34,2013$.

20. Guo XB, Guan S, Fan Y and Song LJ: Local thrombolysis for severe cerebral venous sinus thrombosis. AJNR Am J Neuroradiol 33: 1187-1190, 2012.

21. Goyal M, Menon BK, van Zwam WH, Dippel DW, Mitchell PJ, Demchuk AM, Dávalos A, Majoie CB, van der Lugt A, de Miquel MA, et al: Endovascular thrombectomy after large-vessel ischaemic stroke: A meta-analysis of individual patient data from five randomised trials. Lancet 387: 1723-1731, 2016.

22. Li G, Zeng X, Hussain M, Meng R, Liu Y, Yuan K, Sikharam C, Ding Y, Ling F and Ji X: Safety and validity of mechanical thrombectomy and thrombolysis on severe cerebral venous sinus thrombosis. Neurosurgery 72: 730-738, 2013.

23. Chow K, Gobin YP, Saver J, Kidwell C, Dong P and Viñuela F: Endovascular treatment of dural sinus thrombosis with rheolytic thrombectomy and intra-arterial thrombolysis. Stroke 31: 1420-1425, 2000.

24. Blackham KA: Extensive dural sinus thrombosis: Successful recanalization with thrombolysis and a novel thrombectomy device. J Neurosurg 114: 133-135, 2011.
25. Curtin KR, Shaibani A, Resnick SA, Russell EJ and Simuni T: Rheolytic catheter thrombectomy, balloon angioplasty, and direct recombinant tissue plasminogen activator thrombolysis of dural sinus thrombosis with preexisting hemorrhagic infarctions. AJNR Am J Neuroradiol 25: 1807-1811, 2004.

26. Dowd CF, Malek AM, Phatouros CC and Hemphill JC III: Application of a rheolytic thrombectomy device in the treatment of dural sinus thrombosis: A new technique. AJNR Am J Neuroradiol 20: 568-570, 1999.

27. Chen H, He X, Xie G, Liang J, Ye Y, Deng W, He Z, Liu D, Li D, Liu X and Fan Z: Cardiovascular magnetic resonance black-blood thrombus imaging for the diagnosis of acute deep vein thrombosis at 1.5 Tesla. J Cardiovasc Magn Reson 20: 42, 2018.

28. Siddiqui FM, Dandapat S, Banerjee C, Zuurbier SM, Johnson M, Stam J and Coutinho JM: Mechanical thrombectomy in cerebral venous thrombosis: Systematic review of 185 cases. Stroke 46: 1263-1268, 2015.

29. Scott JA, Pascuzzi RM, Hall PV and Becker GJ: Treatment of dural sinus thrombosis with local urokinase infusion. Case report. J Neurosurg 68: 284-287, 1988.

30. Canhao P, Falcao F and Ferro JM: Thrombolytics for cerebral sinus thrombosis: A systematic review. Cerebrovasc Dis 15: 159-166, 2003.

31. D'Alise MD, Fichtel F and Horowitz M: Sagittal sinus thrombosis following minor head injury treated with continuous urokinase infusion. Surg Neurol 49: 430-435, 1998.

32. Poon CS, Chang JK, Swarnkar A, Johnson MH and Wasenko J: Radiologic diagnosis of cerebral venous thrombosis: Pictorial review. AJR Am J Roentgenol 189 (6 Suppl): S64-S75, 2007.

33. Zuurbier SM, Coutinho JM, Majoie CB, Coert BA, van den Munckhof P and Stam JJ: Decompressive hemicraniectomy in severe cerebral venous thrombosis: A prospective case series. Neurol 259: 1099-1105, 2012.

This work is licensed under a Creative Commons Attribution-NonCommercial-NoDerivatives 4.0 International (CC BY-NC-ND 4.0) License. 